\title{
Relative effectiveness and the European pharmaceutical market
}

\author{
Bengt Jönsson
}

Published online: 26 January 2011

(C) Springer-Verlag 2011

The development of a single European market for medicines over the last decades is a major achievement by European Community legislation. The establishment of the European Medicines Agency (EMA) in 1993 was crucial for the breaking up of system of separate national markets, creating a free flow of medicines throughout the European Union. However, the benefits of a more competitive generics market and from parallel import and export have developed slowly, and there are still potential gains from improved efficiency in the market for generic drugs.

The harmonization of market authorization for new medicines through EMA was a necessary but not sufficient condition for the development of a single market. Pricing and reimbursement measures at the national level also have an impact on the functioning of the internal market. The transparency directive from 1989 is the second important legislation for the development of a European market for medicines. Since Member States have the power to organize and finance their health care systems, this directive is limited to procedural obligations for member states. The directive states that a decision has to be made within 180 days, decisions have to be made using objective and verifiable criteria and there has to be an appropriate appeal mechanism [1].

A major change in the market in the last decade is the increasing importance of health technology assessment (HTA), including cost-effectiveness, for decisions about market access. While HTA has been a health policy instrument in Europe since the 1980s, it is only during the last decade that it has been directly linked to decisions about pricing, reimbursement and guidelines for new

B. Jönsson $(\bowtie)$

Stockholm School of Economics, Stockholm, Sweden

e-mail: Bengt.Jonsson@hhs.se medicines. The influence of national agencies, such as NICE and IQWIG, has been so great that it can be seen as a step back, toward national decisions about the availability of drugs. A new medicine can be marketed, but without inclusion in national reimbursement systems there is a very limited market in practice. The growing number of orphan drugs and targeted therapies aimed at small patient groups and consequently with high prices has contributed to this. European-wide market authorization in itself has no value for the innovator and does not make a single market working if other restrictions persist on the national level.

Since third-party payment for medicines is the fundamental issue, you may argue that the only way to form a single market is to create a single health insurance in Europe. At the moment, patients in Europe have very different health insurance coverage. Health care spending per capita varies with a factor of three, mainly reflecting differences in GDP per capita between EU countries. Health care systems in Europe are converging, but the process is very slow and the relevant question is what can be done now to further develop the European market for medicines. A need for action is also triggered by a third piece of European legislation, the EU directive on patients rights to cross-border health care [2]. The directive is set to clarify the right of patients to seek health care in another EU country while being reimbursed by their national system. It is important for creating a European market for health care and will force health care payers in different countries to harmonize decisions on what they are paying for with their health insurance. A European-wide health insurance is far away, but the need for a policy of harmonization is already here. The key concepts for development of a new policy and potential amendments to legislation to further improve the functioning of the internal market for medicines are assessment of value, access to therapy, and reward for 
innovation. We will examine how relative effectiveness relates to these three concepts.

\section{Relative effectiveness and assessment of added therapeutic value}

Market authorization is based on the concepts efficacy and safety. Pricing and reimbursement decisions at the national level are primarily based on an assessment of cost-effectiveness (CEA), often as part of a more general HTA. Cost-effectiveness is a ratio between the difference in effectiveness, i.e. relative effectiveness, and the difference in costs. It is easy noticeable that assessments of costeffectiveness are, in a majority of cases, driven by the evidence on relative effectiveness rater the differences in costs. For example, negative NICE decision on cancer drugs was, at least until 2006, driven mainly by lack of data on effectiveness. The negative decisions by IQWIG on insulin analogues and statins were in the same way determined by lack of evidence on relative effectiveness. Decisions by the Transparency Commission in France are based on relative effectiveness alone; cost-effectiveness has no formal role in the pricing and reimbursement process. Controversial decisions by TLV, the Swedish pricing and reimbursement agency, have in most cases concerned the interpretation of relative effectiveness based on clinical trial data; for example, Sutent (sunitinib) in MRCC that got a positive decision by a divided board, while Tyverb (lapatinib) in MBC got a negative decision [3].

In cases where price is a decisive factor for the reimbursement decision, it is not the price alone, but the price and relative effectiveness. As Ken Patersson, head of the SMC, The Scottish Medicines Consortium concluded in a review of cancer drugs assessed by SMC: "The new cancer drugs are not too expensive; they are simply not good enough" [4]. Relative effectiveness is thus a key concept for the assessments of value, and thus for pricing and reimbursement.

Since there are no national data on relative effectiveness before a new product has been used in the market, it seems reasonable to challenge the different national interpretations of available data for assessment of relative effectiveness and explore the opportunities for a more harmonized approach. A Working Group on Relative Effectiveness was also established in 2005, within the High Level Pharmaceutical Forum (HLPF), as a response to the recommendations from the G-10 High Level Group on Innovation and Provision of Medicines. Relative effectiveness was considered by the working group as a "complex, though promising area" with the objective to target limited public funds toward most valuable innovative medicines. In their conclusions regarding relative effectiveness, the HLPF "acknowledges the distinction between the scientific assessment of relative effectiveness of medicinal products and health economic assessments of their costs and benefits, and endorses the aim of relative effectiveness assessments to compare healthcare interventions in daily practice and classifying them according to their added therapeutic value" [5].

The recommendation of the HLPF goes along two different routes: one toward the individual Member States and the other toward an increased role for EMA. Member States are encouraged to develop methods for relative effectiveness assessments (REA) and to exchange information about best practices. This duplicates work already ongoing for HTA by INATHA and EUnetHTA and recently REA has been included in the EUnetHTA Joint Action 2010-2012 [6]. The logic of this from a health economics point of view is that the development of methods and data collection for REA and CEA can be done within a coherent HTA framework. The distinction between REA and CEA is not important from a national perspective, and the same agencies will in most cases do both types of studies.

Development of methods and best practices and exchange of information between Member States can contribute to improved efficiency in assessment of health technologies in Europe. But the contribution may be rather small compared to the potential gains from an increased division of labor and mutual recognition of assessments between member states. The work of the Joint Action must also focus on actions that directly can be linked to efficiency improvements, if the efforts should have any impact. One opportunity for improved efficiency in European HTA is in the area of indirect comparisons of effectiveness. Such comparisons are based on available international clinical studies, and it is a waste of resources to undertake these meta-analyses for each Member State (MS). Costs will be lower and quality will be improved if this work is shared among the MS. Another opportunity for improved efficiency comes from the use of a common European instrument for measuring health outcome. Clinical studies have often several outcomes (endpoints) that need to be condensed to a common effectiveness measure to make a REA relevant. Important work has already been done on the development of Euro-QoL (EQ-5D) to take that role [7]. The use of one instrument as a reference does not take away the opportunity to use other effectiveness measures, but without a common measure of effectiveness, relative effectiveness assessment will not fulfill its expectations. A further advantage of this measure is that it also can be used in cost-effectiveness studies, which allow comparisons both within and between therapeutic areas. Disease- or treatment-specific effectiveness measures will not allow this. 


\section{Relative effectiveness and EMA}

The distinction between" the scientific assessment of relative effectiveness of medicinal products and health economic assessments of their costs and benefits" is not important from a HTA perspective, but makes sense if the purpose is to extend the mandate for market authorization. The recommendations of the HLPF also includes a recommendation to "consider how information in the European Public Assessment Report and the National Public Assessment Report, as fore seen in article 13(3)and article 28 of directive 2001/83/EC, can further contribute to relative effectiveness assessment". The current regulation makes it possible to create a role for EMA to collect information on methods that Member States' competent authorities use to determine the added therapeutic value that any new medicinal product provides, with an aim of harmonization. EMA seems in this case to differ from the FDA, where it is difficult to see an option for a similar role at the moment. ${ }^{1}$

But at the same time as HLPF created an opportunity for EMA to play a role in REA, the role was reduced by stating that the specific aim of REA is "to compare health care interventions in daily practice, and classifying them according to their added therapeutic value". The comparative advantage for EMA is in the evaluation of scientific data before market authorization. EMA has also in their contributions to the discussion focused on the distinction between relative efficacy and relative effectiveness [8]. While there may be benefits from a further emphasis on relative efficacy in the EPAR (European Public Assessment Report), this will not be of major importance unless the assessment includes an evaluation of how efficacy in experimental studies can predict effectiveness in clinical practice.

To what extent clinical trials conducted during the development process are good models for REA depend on their design, type of patients enrolled, choice of outcome measures, etc., EMA has since long time built both capacity and competence for such advice based on regulatory criteria. Following earlier initiatives by pharmaceutical companies and national agencies, for example TLV and LMV in Sweden, a first multi-stakeholder consultation was also held at EMA in October 2010 [9]. The three pilot consultations are organized by a consultancy firm, and it is difficult to predict how this initiative will develop for the future and to what extent it will bridge the gap between efficacy and effectiveness [10].

\footnotetext{
${ }^{1}$ However, FDA and the Centers for Medicare and Medicaid Services (CMS) are considering establishing a process for overlapping evaluations of premarket, FDA-regulated medical products sponsor and both agencies agree to such parallel review. Federal Register, Vol 75 No 180: 57045, 2010.
}

EMA also have an extensive program for the assessment of risk benefit, including the collection of data after market authorization. You may argue that through the assessment of benefit-risk EMA is already involved in REA and added therapeutic value. With an increasing focus in drug development on orphan drugs and targeted therapies, the assessment of risk will also change. The major problem with uncertainty for expensive drugs used in small populations of patients is not the risk of health loss, but rather the risk of waste of money if the medicine does not work. It is different with low priced and/or non-reimbursed medicines used by large populations, where the health risk is dominating. From the perspective of the health economist, it is a small step to include cost in the benefit-risk assessment models.

Uncertainties about risk and effectiveness of new medicines are similar and can only be reduced by further studies and data. First there is a choice between what should be requested before the product is used in clinical practice, and second what should be collected in follow up studies in clinical practice. A greater influence of payers and other stakeholders such as patients on the development process will change the trade-offs, with important implications for the drug development process. It is difficult to see how economic aspects, including cost-effectiveness can be kept out of this. To this, we can add the potential savings that can be made if data on risks, effects, and resource utilization can be collected within a comprehensive framework. For rare diseases, and with the increase in targeted therapies these will be the rule rather than the exception, a European collaboration for data collection also make sense from both an economic and a public health perspective. During their EU presidency in 2009, Sweden also took an initiative of a pilot project to gain experience how this can be done [11].

While at present the EMA is not in the driving seat, it is difficult to see any other role for the future than a full engagement in the issues described above [12]. The "Road Map to 2015", adopted by the management board in December 2010, also includes initiatives increase the role of EMA to provide information on relative efficacy/effectiveness. If the EMA is not given the mandate for this, its role will be marginalized and other institutions will be developed to take its place. This would be unwise, expensive, and take long time, and in these matters, time is both money and health.

\section{Relative effectiveness and variations in access to medicines}

A correct assessment of value is important for an efficient market for medicines. Resources should be allocated to the 
medicines that give most health for the population served. The persistence of large variations in the use of medicines or surgical procedures indicates that there are opportunities for improvements. Early studies of "small area variations" documented large variations within a country, which were attributed to availability of resources and discretionary decision making by doctors. For interventions with clearly defined indications the variation was less. Variations in use of medicines between countries is hardly surprising when markets a national, but in the 1970s there was a debate about "the drug lag" between United States and Europe, based in differences in regulatory decision regarding for example cardiovascular drugs. For an excellent economic analysis of regulatory decision making related to this, pointing out the unavoidable trade-offs, see Pelzman 1973 [13].

With the development of the internal market for medicines, country variations in use of therapies that are targeted to specific patient populations have become an issue for health policy. New cancer drugs have been an area for comparative studies, as well as new biological for the treatment of RA and MS [14]. These studies have shown that there are large variations in use also between countries which have comparable health care expenditures $[15,16]$. One important determination of these variations seems to be different interpretations of relative and cost-effectiveness by pricing and reimbursement agencies. This raises the question to what extent relative effectiveness varies between countries, since such differences would be legitimate and not an indicator of imperfection in the market.

There are three types of potential sources of variation in relative effectiveness between countries.

- The first are differences in the target population for the drug. The number of potential patients determined by incidence and severity of disease may differ. For example, the risk related to osteoporosis is higher in Northern than Southern Europe, which means that the absolute risk reduction, which determines value, is larger and you would expect a higher number of treated patients. The extent and importance of these variations for differences in effectiveness of a drug between countries could be documented and analyzed if the relevant data are collected and analyzed, which is not the case at the moment. It should also be observed that these differences in relative effectiveness do not change the cost-effectiveness of the drug in a defined population; it merely influences how many patients should be treated at a defined threshold and thus the total value of the drug for the country.

- The second source of variations in relative effectiveness of medicines is national variations in what constitutes the relevant comparator. Clinical practice patterns are defined by a number of factors, including organization, availability of resources and economic factors. Often there are not only one relevant alternative making it necessary to carry out several studies of relative effectiveness The choice of a comparator is further complicated by the fact that the relevant comparison in practice often is between treatment strategies rather than between products. We do not know the magnitude of differences in relative effectiveness due to clinical practice variations, and studies are needed. However, you would expect that this source of variation between countries will diminish over time due to convergence of health care systems and a more rational use of medical technology.

- A third source of variation in effectiveness and thus value are differences in productivity between countries and health care systems. The effectiveness of a specific medicine may be dependent on how it is combined with other health inputs. Efficiency is not only about doing the right things, but also doing things right. A common complaint about economic evaluations, which includes assessment of relative effectiveness based on clinical trial or best practice data, is that they do not take into account that new medicines enters into an imperfect world.

Health care systems are increasingly allocating resources based on outcome rather than input. For new medicines we see reimbursement and market access conditioned upon indicators of outcome [17]. It may only be that data about patient characteristics are requested to satisfy that the drug is correctly used, but there are also examples where payment is related to response or even final outcome. If this trend continues, as it probably will, it will dramatically change the market and make providers of medicines jointly responsible for delivery of services. Cost-effectiveness analysis will increasingly be based on data from actual clinical practice, rather than modeling based on clinical trial data. Such models will however be important to guide the development process and for the design of clinical practice studies. Studies of variations in access to new medicines based on individual patients data rather than sales statistics will be important in health policy as benchmarks for both efficiency and equity.

\section{Relative effectiveness and reward for innovation}

It is difficult to understand the interest of reimbursement and HTA agencies in REA without reference to how pharmaceutical innovation has been rewarded during the last decades. New medicines have created large social gains, of which the innovators only have been able to 
capture a very small part. Examples of this are the drugs for treatment of aids and statins for reduction of cardiovascular events [18, 19]. The producer surplus (profits) on the Swedish market was estimated at $2-5 \%$ of the total social surplus (value) created by the use of statins, which is comparable to the $7 \%$ estimated for HIV drugs.

But members of reimbursement committees also observe a number of new drugs coming to the market with rather marginal benefits, asking for and receiving premium prices. Incremental innovations are the rule and break through innovations are the exception, and it may very well have been the case that the market has rewarded the real innovations too little and the incremental innovations too much in the past. Can REA change this? One answer to this question is that change is already under way. But drug development is a long process and it takes time to see the changes in terms of the new drugs coming to the market. The present focus on REA can thus be seen as a confirmation that changes are under way.

REA has one important advantage from a pricing and reimbursement policy based on reference pricing. If there is no documented improvement in effectiveness, there will not be any discussion about a premium price. The new medicine can be clustered together with other medicines in a group. Arguments about a new mechanism of action or advantages shown in clinical trials can be put aside if they cannot be translated in a credible way to improved effectiveness in clinical practice. This is a strong argument for the innovative pharmaceutical industry to either direct the efforts to develop drugs that can be launched at a competitive price on an established market, or drugs for a new market where a monopoly position can be gained at least for some time. The downside of this is that while it increases the potential rewards, it also increases the risk. HTA is a superior strategy for obtaining value for money because it addresses not only price, but also the appropriate indications for the use of the drug and the relation between additional value and additional costs. The latter is important to provide correct incentives for investments in pharmaceutical R and D [20].

REA may be useful as an instrument for changing the rewards for innovation on the European market for medicines, but additional policies may be needed to make it work. Since risks and costs of drug development are increasing, there is a need that the health care systems clearly communicate what they are willing to pay for. We may also increasingly observe that if health care systems have clear demands on what type of innovations should be developed, they may also have to contribute directly or indirectly to the development process. One way of doing this could be to take a greater part of the costs for postlaunch studies, but perhaps also for development of information systems that reduce the costs for clinical trials. In the end, it comes to the prices paid for innovations and how fast patients can get access to the new drugs. To make those decisions, REA are not enough, and we need the information from social cost benefit studies to guide those decisions.

\section{Relative effectiveness and cost-effectiveness}

Relative effectiveness has the advantage over cost-effectiveness that it does not include the word cost. In the United States where the concept and methods for HTA were invented and developed but never much used in health policy, the new term comparative effectiveness research (CER) has taken its place [21]. A new institute for CER has been established: the non-profit Patient-Centered Outcomes Research Institute (PCORI) to set priorities and develop methods for CER. An interesting aspect of the legislation, the Patient Protection, and Affordable Care act (ACA) is that PCORI is prohibited from developing or using a cost-per-QALY threshold. We have no legislation against the use of cost-effectiveness information in Europe, but policy makers seem to share the same ignorance or political posturing [22, 23]. Why is it so difficult, also in Europe, to accept that comparisons of cost-effectiveness ratios using for example a cost per QALY metric can be useful information for a decision? It does not prevent any decision maker to take other factors into account. Neither does it prevent research on the implication of using such a metric, for example if it discriminates on factors such as age or disability. Such research could also stimulate the development of alternative measures. It would reduce the opportunity to continue ignoring the necessary trade-offs and thus persisting with non-transparent decisions that prevent the directing resources toward activities that give the greatest health gains. This would be welcome particularly when we are heading toward a situation of crisis in public finances which will force us to make even more difficult priorities. It may be the case that the medical public policy experts who today inform reimbursement decisions in most countries feel threatened by "economic assessment" and that explains the resistance [24]. But the challenge comes from health economics, not necessarily from health economists. The important task of developing relevant and credible information for decisions about new medical technologies are multidisciplinary and best undertaken as a team work where different competences contribute.

Relative effectiveness assessment is promising but complex, but it will be more promising and less complex if it is linked up with cost-effectiveness. Methods for assessing relative effectiveness within a framework of costeffectiveness are well established, and there is a wealth of 
knowledge, experience, and competence to build on. Decisions about the use of health care resources in Europe are national, regional, local, and individual. But this should not prevent EU for the development of policy and legislation that give decision makers the relevant information for their decisions, if it is good for both our health and wealth.

\section{References}

1. Council Directive 89/105/EEC

2. Proposal for a Directive of the European Parliament and the Council on the application of patients rights in cross-border health care. Brussels 2.7.2008, COM (2008) 414 final

3. The decisions are available at www.tvl.se. 080915-tyverb and 061160-sutent

4. Report from HCDMF Stockholm 2009

5. http://ec.europa.eu/pharmaforum/

6. http://www.eunethta.net/Public/Work_Packages/EUnetHTAJoint-Action-2010-12/

7. http://www.euroqol.org/

8. Eichler, H.G., Bloechl-Daum, B., Abadie, E., Barnett, D., König, F., Pearson, F.: Relative efficacy of drugs: an emerging issue between regulatory agencies and third-party payers. Nature reviews. Drug Discov. 9, 277-290 (2010)

9. www.ema.europa.eu/news

10. www.tapestrynetworks.com

11. http://www.se2009.eu

12. Jönsson, B.: Health technology assessment: Regulators or payers-who will take the lead? Clin. Therapeut. 30(5), 1-4 (2008)
13. Pelzman, S.: An evaluation of consumer protection legislation: The 1962 amendments. J. Political Econ. 81, 1049-1091 (1973)

14. www.comparatorreports.se

15. Jönsson, B., Wilking, N.: A global comparison regarding patient access to cancer drugs. Ann. Oncol. 18(suppl3), 1-75 (2007)

16. Extent and causes of international variation in drug usage. A Report for the Secretary of State for Health by Professor Sir Mike Richards CBE, (July 2010)

17. Carlson, J.J., Sullivan, S.D., Garrison, L.P., Neumann, P.J., Veenstra, D.L.: Linking payment to health outcomes: A taxonomy and examination of performance-based reimbursement schemes between health care payers and manufacturers. Health Policy 96(3), 179-190 (2010)

18. Philipson, T., Jena, A.B.: Who Benefits from New Medical Technologies? Estimates of Consumer and Producer Surpluses for HIV/AIDS Drugs. Forum for Health Economics \& Policy 9(2), (2006) (See also Philipson T and Jena A B : Innovation and technology Adoption in Health Care Markets. The AIE Press, Washington DC (2008))

19. Lindgren, P., Jönsson, B.: Cost-effectiveness of statins over time. European J. Health Econ. (submitted)

20. Drummond, M., Jönsson, B., Rutten, F., Stargardt, T.: Reimbursement of Pharmaceuticals: Reference Pricing versus Health Technology Assessment. EJHE (2010) Forthcoming

21. Tunis, S.R., Benner, J., McClellan, M.: Comparative effectiveness research: Methods development, policy context, and research infrastructure. Stat. Med. 29(19), 1963-1976 (2010)

22. Shaw, J.W.: Lack of support for cost-utility analysis in the health care reform law a case of ignorance or political posturing? Clin. Therapeut. 32, 1091-1092 (2010)

23. Neumann, P.J., Weinstein, M.C.: Legislating against use of costeffectiveness information. NEJM 363(16), 1495-1497 (2010)

24. Pouvourville, G.: A French approach to cost-effectiveness analysis? Eur. J. Health Econ. 11, 521-523 (2010) 